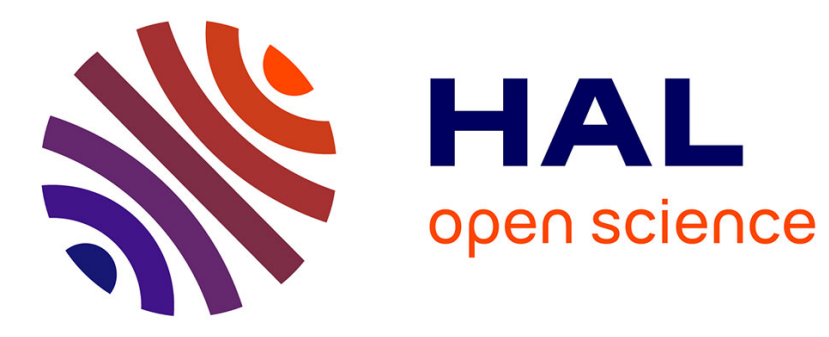

\title{
Design of a navigation filter by analysis of local observability
}

Pierre-Jean Bristeau, Nicolas Petit, Laurent Praly

\section{To cite this version:}

Pierre-Jean Bristeau, Nicolas Petit, Laurent Praly. Design of a navigation filter by analysis of local observability. 49th IEEE Conference on Decision and Control, Dec 2010, Atlanta, United States. pp.1298-1305, 10.1109/CDC.2010.5717848 . hal-00548941

HAL Id: hal-00548941

https://hal-mines-paristech.archives-ouvertes.fr/hal-00548941

Submitted on 20 Dec 2010

HAL is a multi-disciplinary open access archive for the deposit and dissemination of scientific research documents, whether they are published or not. The documents may come from teaching and research institutions in France or abroad, or from public or private research centers.
L'archive ouverte pluridisciplinaire HAL, est destinée au dépôt et à la diffusion de documents scientifiques de niveau recherche, publiés ou non, émanant des établissements d'enseignement et de recherche français ou étrangers, des laboratoires publics ou privés. 


\title{
Design of a navigation filter by analysis of local observability
}

\author{
Pierre-Jean Bristeau, Nicolas Petit, Laurent Praly
}

\begin{abstract}
This paper presents an inertial navigation filter designed for an automotive vehicle not equipped with any GPS receiver. The task of this filter is to provide relative position information over a relatively long period of time (tens of minutes). The filter consists of several partial state observers that, one after another, reconstruct key information for the whole state estimation. The observer relies on a sufficient condition to guarantee uniform complete observability of a general bounded linear time-varying system using (point-wise) differential rank conditions. From this condition, we construct a collection of filters well-suited for each possible trajectory of the vehicle. This results in temporally interconnected observers which are of the Kalman filter type. It is proven that each of them asymptotically converges to zero. We illustrate this design with trajectory estimation obtained on simulation data. Finally, experimental results using low-cost sensors show the potential and the relevance of the approach.
\end{abstract}

\section{INTRODUCTION}

In this paper, we expose the design of a navigation filter for an automotive vehicle not equipped with any GPS receiver. Among others, low-cost inertial sensors (MEMS) and a velocimeter are used to determine, through a data fusion algorithm, relative positioning information over a relatively long period of time (tens of minutes). The originality of the approach is to propose an observer specifically designed to take advantage of well-known properties of the trajectory and the dynamics of the vehicle under consideration.

Inertial navigation is a well-established technique which has taken key roles in the aerospace industries [1][2], as well in other areas such as undersea navigation or dynamic positioning systems [3]. It has also recently emerged as an enabling technology under the forms of MEMS sensors in numerous low-cost applications (small UAVs [4][5], ground robotics, cell-phones, among others). Yet, its main limitation is the unavoidable drift of the estimates [1]. The culprits are the biases of the sensors, mostly accelerometers and gyroscopes, which result in drift in velocity and, consequently, in position estimates [6]. With MEMS sensors, those drift appear over short time periods (tens of seconds [7]). This usually discards them for most critical applications if they are not complemented by some other source of information.

A key feature of the setup we consider in this paper is the availability of a velocimeter which provides a relatively dependable estimate of the vehicle body velocity. This sensor is usually available in most vehicles today. It can be obtained

P.J. Bristeau is $\mathrm{PhD}$ candidate in Mathematics and Control, CAS, Unité Mathématiques et Systèmes, MINES ParisTech, 60 bvd Saint-Michel, 75272 Paris, FRANCE pierre-jean.bristeau@mines-paristech. fr

N. Petit and L. Praly are with the CAS, Unité Mathématiques et Systèmes, MINES ParisTech, 60 bvd Saint-Michel, 75272 Paris, FRANCE through various sensor technologies application (e.g. Doppler radar [8], camera [9], Pitot tube). Each technology has its own flaws and advantages [10]. Yet, this scalar information is not sufficient to estimate the motion of the vehicle as rotations come into play. Gyroscopes can be used to determine rate-of-turn information but they also have (non-constant) biases which are causes of substantial drifts.

The contribution of this paper is the design of a navigation filter which cancels these drifts in the sense that the sources are identified and estimated on-board. The proposed design is based on an analysis of observability along various possible trajectories of the system. These investigations serve to prove convergence of a collection of Kalman filters used to estimate the states of linear time-varying (LTV) dynamics.

Usually, the convergence of the Kalman filter is proven under the assumption of Uniform Complete Observability (UCO). Yet, from its definition, the UCO property is difficult to establish since it calls for the computation of the observability Grammian, and so, of integrals involving the transition matrix.

We would like to have at our disposal a differential condition, similar to the usual Kalman rank criterion for linear time-invariant (LTI) systems. In the literature [11][12][13][14], some prospects have been proposed, but without equivalence to UCO property. We will propose a sufficient condition based on differential observability.

In the case under study, the trajectory involves various dynamics and measurement equations depending whether straight-line motion or curve motion is under consideration. As will be demonstrated, the global problem of full-state estimation can be handled by sequentially estimating subsets of the full state, each one being estimated during an appropriate part of the trajectory.

We consider the vehicle as a six degrees of freedom (DOF) rigid body moving without sideslip. The vehicle is characterized by a tridimensional position, a curvilinear velocity and three attitude angles. We assume it is equipped with a velocimeter, a trihedron of accelerometers, a trihedron of gyroscopes, and an altimeter. Certainly, these sensors are not sufficient to get the absolute position and heading of the vehicle, but this is not our goal. Only relative positioning is desired here.

The work presented in this article is inspired by the observers interconnection theory (see [15]). Separately but simultaneously, we estimate the velocity, the angular dynamics (angles, rates and biases), and finally, the accelerations (and the biases associated to the sensors). The main difficulty is to estimate the angular dynamics. For this task, we propose temporally interconnected observers (TIO) arguing 
that during straight-line motion, one can estimate the pitch dynamics, while, during curve motion, it is possible to catch the roll dynamics. Yaw bias can be estimated at rest. From these angles, one can estimate the accelerometers biases, so that accelerometers can be used to exert fault detection on the velocimeter.

The paper is organized as follows. In Part II, we recall some definitions of observability, along with ways to establish this property. We give a sufficient condition for UCO and recall that it guarantees the convergence of Kalman filters. In Part III, we detail the problem under consideration and present the TIO designed for this problem. The merits of this approach are illustrated with simulation results. In Part IV, we present results from an actual implementation on board an automotive system.

\section{A CONDITION FOR UNIFORM COMPLETE OBSERVABILITY}

We start with some recall on UCO. Consider a linear timevarying (LTV) plant, with $A(t), B(t), C(t)$ analytic:

$$
\left\{\begin{array}{l}
\dot{x}(t)=A(t) x(t)+B(t) u(t) \\
y(t)=C(t) x(t)
\end{array}\right.
$$

For now, the (very general) problem under consideration is the asymptotic reconstruction of the state from the knowledge of $A(t), B(t), u(t), C(t)$ and $y(t)$.

\section{A. Complete Observability and Uniform Observability}

The previously mentioned observation task can be performed using a Kalman filter (others choices are possible using time-varying observation gains, see e.g. [16][17][18][19]). The convergence of the Kalman filter can be proven using some observability properties of the system (1). As is well known (see e.g. [14]), point-wise observability is usually not sufficient, and in fact, UCO property has to be established. This property follows from the complete observability ( $\mathrm{CO}$ ) and the uniform observability (UO) recalled below for convenience.

Note $\Phi(s, t)$ the transition matrix associated to A.

$$
\frac{\partial \Phi}{\partial t}(t, s)=A(t) \Phi(t, s) \quad \Phi(t, t)=I
$$

Definition 1: [20] The system (1) is $C O$ if and only if every present state $x(t)$ can be determined when $A(s)$ and $C(s)$ and $y(s)$ for $s \in\left(t_{0}, t\right)$ are known for some $t_{0}(t)<t$.

Theorem 1: [20] The system (1) is $C O$ if and only if for every $t$ there exists a $t_{0}(t)<t$ such that

$$
W^{*}\left(t_{0}, t\right)=\int_{t_{0}}^{t} \Phi^{T}(s, t) C^{T}(s) C(s) \Phi(s, t) d s
$$

is positive definite. The application $W^{*}\left(t_{0}, t\right)$ is the reconstructibility Grammian.

Definition 2: [20] The system (1) is $U O$ if and only if there exists $\gamma, \delta, \sigma$ so that for every $t, W^{*}(t-\sigma, t)$ is positive definite and

$$
0<\gamma I \leq W^{*}(t-\sigma, t) \leq \delta I
$$

The discussed UCO property is defined below.

Definition 3: [20][21] The system (1) is UCO if the following relations hold for all $t$ :

$$
\text { (i) } 0<\alpha_{0}(\sigma) I \leq W^{*}(t-\sigma, t) \leq \alpha_{1}(\sigma) I
$$

$0<\beta_{0}(\sigma) I \leq \Phi^{T}(t-\sigma, t) W^{*}(t-\sigma, t) \Phi(t-\sigma, t) \leq \beta_{1}(\sigma) I$

where $\sigma$ is a fixed constant .

In the case of bounded matrices, the following theorem provides a simpler necessary and sufficient condition.

Theorem 2: [22][23] A bounded system $[A(t), B(t), C(t)]$ is $U C O$ if and only if there exists $\sigma>0$ such that for all $t$,

$$
W^{*}(t-\sigma, t) \geq \alpha_{0}(\sigma) I>0
$$

Determining whether the uniform lower boundedness of $W^{*}(t-\sigma, t)$ holds is usually considered as a very difficult task. In general, computing the transition matrix is involved and computing $W^{*}(t-\sigma, t)$ is hardly tractable. Much more conveniently, a point-wise investigation of the observability of the analytic system (1) can yield interesting conclusions.

\section{B. Differential Observability}

In [12], the possibility to establish observability from the study of the observability matrix defined below has been investigated. A theorem exposing a rank condition to prove the $\mathrm{CO}$ on an interval (see also [24]) is as follows.

Definition 4: [12] The observability matrix $Q_{o}(t)$ is defined below, where $n$ is the dimension of $x$ :

$$
\left\{\begin{array}{l}
Q_{o}(t)=\left[\begin{array}{llll}
Q_{0}(t) & Q_{1}(t) & \ldots & Q_{n-1}(t)
\end{array}\right] \\
Q_{0}(t)=C^{T}(t) \\
Q_{i+1}(t)=\dot{Q}_{i}(t)+A^{T}(t) Q_{i}(t)
\end{array}\right.
$$

Theorem 3: [12] The system (1) is $C O$ on the interval $\left(t_{0}, t_{1}\right)$ if $Q_{o}(t)$ has rank $n$ for some $t \in\left(t_{0}, t_{1}\right)$.

Interestingly, in the same paper [12], the notion of UO on an interval is also considered: the difficulty to find an uniform (independent of the time) bound for the observability Grammian is alleviated by the knowledge of bounds on the time.

Definition 5: [12] The system (1) is said to be $U O$ on the interval $\left(t_{0}, t_{1}\right)$ if $Q_{o}(t)$ has rank $n$ for all $t \in\left(t_{0}, t_{1}\right)$.

We generalize this approach by considering the following sufficient condition for UCO (in the sense of Definition 3).

Theorem 4: The bounded system (1) is UCO if there exists $\mu>0, m \in \mathbb{N}$ such that for all $t$ :

$$
\mathbb{O}(t)=\left(\begin{array}{lll}
Q_{0}(t) & \ldots & Q_{m}(t)
\end{array}\right)\left(\begin{array}{c}
Q_{0}^{T}(t) \\
\vdots \\
Q_{m}^{T}(t)
\end{array}\right) \geq \mu I>0
$$

The proof of this "folk" result is in the appendix because we were not able to give a reference containing a complete proof, and to make the paper self-contained.

\section{Existence of an observer}

Classically, the UCO property serves to guarantee the convergence of Kalman filters.

Theorem 5: [20][25][26] If system (1) is UCO, then there exists an observer of the form:

$$
\dot{\hat{x}}(t)=A(t) \hat{x}(t)+B(t) u(t)-K(t)(C(t) \hat{x}(t)-y(t))
$$


with $K(t)$ given by:

$$
\begin{aligned}
\dot{M}(t)= & A(t) M(t)+M(t) A^{T}(t)-M(t) C^{T}(t) W^{-1} C(t) M(t) \\
& +V+\delta M(t) \\
M(0)= & M_{0}=M_{0}^{T}>0, \quad W=W^{T}>0, \\
K(t)= & M(t) C^{T}(t) W^{-1}
\end{aligned}
$$

with either $\delta>2\|A(t)\|$ for all $t$, or $V=V^{T}>0$.

Now that we have an easy to check condition (2) sufficient to guarantee the convergence of Kalman filters for LTV systems, we move forward to our application.

\section{A NEW USE OF INTERCONNECTED OBSERVERS}

\section{A. Description of the vehicle navigation problem}

The vehicle under consideration in this paper is equipped with a velocimeter, three accelerometers, three gyroscopes and an altimeter. The velocimeter measures the velocity of the vehicle. There is no sideslip. From time to time, the value given by the velocimeter is totally erroneous. The accelerometers and the gyroscopes are biased. The inputs of the driver are unknown.

The variable $u$ denotes the longitudinal velocity of the vehicle, $a_{x}, a_{y}, a_{z}$ the specific accelerations along the body axis, $p, q, r$ the rotation speeds, $\phi, \theta$ the roll and pitch angles and $z$ the altitude (Fig. 1). The subscript ${ }_{m}$ indicates the corresponding measurement and $b_{a_{x}}, b_{a_{y}}, b_{a_{z}}$ (resp. $b_{p}, b_{q}, b_{r}$ ) are the bias of the accelerometers (resp. gyroscopes) which are varying over time. The complete state to be estimated is

$$
X=\left(\begin{array}{ccccccccc}
u & z & b_{a_{x}} & b_{a_{y}} & b_{a_{z}} & a_{x} & a_{y} & a_{z} & \ldots \\
\ldots & \phi & \theta & b_{p} & b_{q} & b_{r} & p & q & r
\end{array}\right)^{T}
$$

The dynamics and measurement equations of the 6 DOF rigid body are derived under the assumptions of zero sideslip, and small angles of roll and pitch. ${ }^{1}$ The dynamics of the accelerations and of the rotation speeds are unknown. The only dependable differential equations that can be written are

$$
\begin{cases}\dot{u}(t)=a_{x}(t)-g \theta(t) & \dot{z}(t)=-u(t) \theta(t) \\ \dot{\phi}(t)=p(t)+r(t) \theta(t) & \dot{\theta}(t)=q(t)-r(t) \phi(t)\end{cases}
$$

\footnotetext{
${ }^{1}$ The yaw angle $\psi$ does not appear since it is unobservable. Nevertheless, all the improvements which are realized on the estimation of the yaw rate are beneficial for the subsequent integration process since $\psi(t)=r(t)+q(t) \phi(t)$.
}

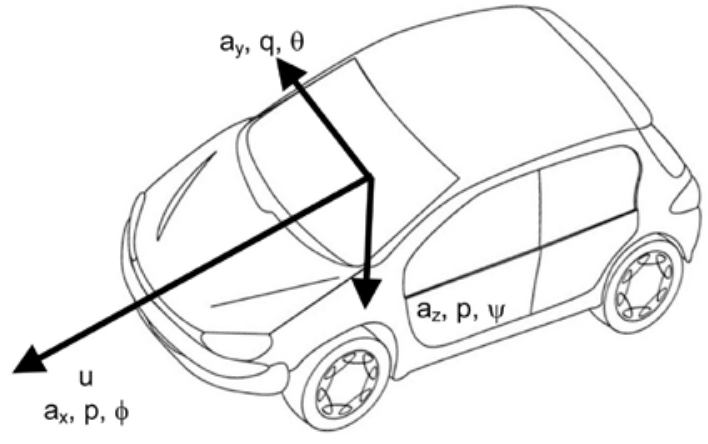

Fig. 1. Notations in the body frame
The bias dynamics are unknown. One could model them with first order dynamics driven by white noise or other pink noise models. From now-on, the considered bias dynamics is the one which contains the less information: bias are assumed constant in the deterministic part of the modeling and unknown inputs are allowed (denoted by $*$ ).

Finally, the measurement equations are

$$
\begin{cases}u_{m}(t)=u(t) & z_{m}(t)=z(t) \\ a_{x_{m}}(t)=a_{x}(t)+b_{a_{x}}(t) & \\ a_{y_{m}}(t)=a_{y}(t)+b_{a_{y}}(t)= & r(t) u(t)-g \phi(t)+b_{a_{y}}(t) \\ a_{z_{m}}(t)=a_{z}(t)+b_{a_{z}}(t)=-q(t) u(t)-g+b_{a_{z}}(t) \\ p_{m}(t)=p(t)+b_{p}(t) \\ q_{m}(t)=q(t)+b_{q}(t) \\ r_{m}(t)=r(t)+b_{r}(t)\end{cases}
$$

\section{B. Observers for Interconnected Systems}

The method we propose to estimate the state is inspired by the theory of observers for interconnected systems widely detailed in [15]. The problem is split into three sub-problems. First, we assume that the velocimeter is well functioning and a velocity observer is directly derived from it (first problem). Considering the velocity as a known parameter, a second problem is to estimate the biases of the gyroscopes, the pitch and roll angles, the corresponding rate of turn and the altitude. Finally, we assume that all the preceding variables are known parameters, and we reconstruct the acceleration and the accelerometers biases (third problem). This proposed observer design corresponds to the interconnection pictured in Fig. 2. The three problems detailed above corresponds to subsystems $\Sigma_{I}, \Sigma_{I I}$ and $\Sigma_{I I I}$ describing respectively the velocity dynamics, the angular dynamics, and the acceleration measurement equations.

It is not literally a cascade interconnection since at last, the accelerometers will be used to detect potentially erroneous information from the velocimeter and, if need be, integrated to estimate the correct velocity. This supplementary interaction (not modeled in the equations (4-5)) is detailed in Fig. 3.

Establishing separately (i.e. when the parameters are known) the observability of the velocity and the observability of accelerometers biases (first and third problems) is trivial, so we now focus on the second problem for which we propose to outline another level of interconnection which suggests an observer design. This point is illustrated in Fig. 3. In the following paragraph, we show how to estimate in a sequence the reduced state

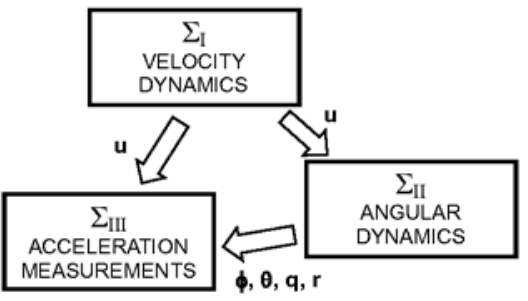

Fig. 2. Observers interconnection 


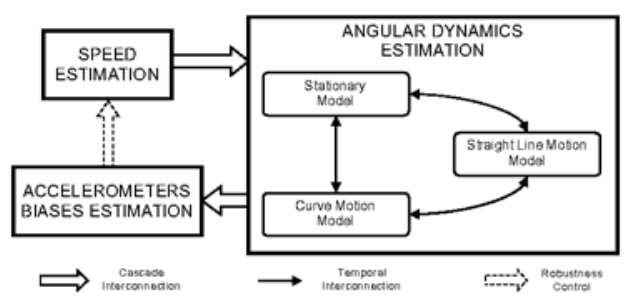

Fig. 3. Two levels of interconnected observers

$X_{I I}=\left(\begin{array}{lllllllll}z & \phi & \theta & b_{p} & b_{q} & b_{r} & p & q & r\end{array}\right)^{T}$ of $\Sigma_{I I}$, considering $u$ as given.

\section{Temporally Interconnected Observers}

In this part, we study three main types of trajectory for the vehicle. These are defined as follows. If the velocity is null, the vehicle is at rest. Otherwise, if the yaw rate is null, the vehicle is going in straight line, else the vehicle is in a curve. As will appear, for each trajectory, a subset of $X_{I I}$ is observable. We note $t_{0}$ the time of switch between two types of trajectory.

1) Stationary Model:

We first consider the vehicle at rest, the speeds are null, the altitude is measured and the angles are unobservable but remain unchanged. The following relations hold.

$$
\left\{\begin{array}{lll}
u(t)=0 & z(t)=z_{m}(t)=z\left(t_{0}\right) & \\
p(t)=0 & q(t)=0 & r(t)=0 \\
\phi(t)=\phi\left(t_{0}\right) & \theta(t)=\theta\left(t_{0}\right) &
\end{array}\right.
$$

The gyroscopes directly provide the values of the biases, and we consider null rates measurements

$$
\begin{aligned}
X_{0} & =\left(\begin{array}{lllllll}
z & b_{p} & b_{q} & b_{r} & p & q & r
\end{array}\right)^{T} \\
\dot{X}_{0} & =\left(\begin{array}{lllllll}
0 & * & * & * & 0 & 0 & 0
\end{array}\right)^{T}, \quad Y_{0}=X_{0}
\end{aligned}
$$

The subset $X_{0}$ is observable and if the angles were already known before the stop of the vehicle, $X_{I I}$ could be estimated at rest.

\section{2) Straight-Line Motion Model:}

Now, we consider the vehicle in straight-line motion $\left(r(t)=0, u(t) \geq u_{m}>0\right)$. The particularity of this trajectory is that the pitch dynamics and the roll dynamics are decoupled. During this phase, the whole pitch dynamics is observable. We now show why.

Consider the subset of variables $X_{1}$ and its dynamics and measurement equations.

$$
\begin{aligned}
X_{1}(t) & =\left(\begin{array}{llll}
z(t) & \theta(t) & b_{q}(t) & q(t)
\end{array}\right)^{T} \\
\dot{X}_{1}(t) & =\left(\begin{array}{cccc}
0 & u(t) & 0 & 0 \\
0 & 0 & 0 & 1 \\
0 & 0 & 0 & 0 \\
0 & 0 & 0 & 0
\end{array}\right) X_{1}+\left(\begin{array}{l}
0 \\
0 \\
* \\
*
\end{array}\right) \\
Y_{1}(t) & =\left(\begin{array}{llll}
1 & 0 & 0 & 0 \\
0 & 0 & 1 & 1
\end{array}\right) X_{1}(t)
\end{aligned}
$$

The maximum velocity and acceleration of the vehicle are bounded. We have, for all $t$,

$$
u_{m} \leq|u(t)| \leq u_{M}, \quad|\dot{u}(t)| \leq \dot{u}_{M}
$$

The local observability matrix (2) is

$$
\begin{array}{r}
\mathbb{O}(t)=\left(\begin{array}{llll}
Q_{0}(t) & Q_{1}(t) & Q_{2}(t)
\end{array}\right)\left(\begin{array}{l}
Q_{0}^{T}(t) \\
Q_{1}^{T}(t) \\
Q_{2}^{T}(t)
\end{array}\right) \\
=\left(\begin{array}{cccc}
1 & 0 & 0 & 0 \\
0 & u^{2}(t)+\dot{u}^{2}(t) & 0 & u(t) \dot{u}(t) \\
0 & 0 & 1 & 1 \\
0 & u(t) \dot{u}(t) & 1 & 1+u^{2}(t)
\end{array}\right)
\end{array}
$$

We wish to prove that there exists $\mu>0$ such that $\mathbb{O}(t) \geq \mu I$ for all $t$. The matrix $(\mathbb{O}(t)-\mu I)$ is symmetric real, therefore it is positive semidefinite if and only if its eigenvalues are positive. Equivalently, we wish to prove that there exists $\mu>0$ such that the eigenvalues of $\mathbb{O}(t)$ are greater than $\mu$ for all $t$. Since $\mathbb{O}(t)$ is symmetric real, these eigenvalues are real and positive. They are the roots of the characteristic polynomial $P(s, t)=|\mathbb{O}(t)-s I|$ which can be lower-bounded, uniformly in $t$, for $s$ positive, by the polynomial $P_{0}(s)$ thanks to Eq. (12)

$$
P_{0}(s)=(1-s)\left(\begin{array}{c}
-s^{3}+2\left(u_{m}^{2}+1\right) s^{2} \\
-\left(u_{M}^{4}+3 u_{M}^{2}+2 \dot{u}_{M}^{2}\right) s+u_{m}^{4}
\end{array}\right)
$$

Yet, $P_{0}(0)=u_{m}^{4}>0$. Therefore, there exists $\mu>0$ such that $P_{0}(s)>0$ for $s \in[0, \mu]$. In turn, the roots of $P(s, t)$ are uniformly bounded by $\mu$ which yields the desired conclusion.

From Theorem 4, the system (9-11) is UCO and one can use a Kalman filter (thanks to Theorem 5) to estimate the subset of variables $X_{1}$. Meanwhile, values for roll and yaw biases are kept constant and the roll angle is just integrated from the corrected roll rate. Consequently, the state $X_{I I}$ is updated by the following equations

$$
\left\{\begin{array}{l}
\dot{\phi}(t)=p(t) \\
p(t)=p_{m}(t)-b_{p}\left(t_{0}\right) \quad r(t)=r_{m}(t)-b_{r}\left(t_{0}\right)
\end{array}\right.
$$

3) Curve Motion Model:

Now, we consider the vehicle in (not necessarily uniform) curve motion. The yaw rate satisfies $|r(t)|>r_{m}$ and coupling between roll and pitch dynamics appears. Together, the two dynamics are unobservable, but, since pitch dynamics is observed during straight-line motion, the pitch bias is kept constant at this estimated value during this curve motion. Then, the roll dynamics can be estimated. The subset of variables $X_{2}$ under consideration is the following

$$
X_{2}(t)=\left(z(t) \quad \phi(t) \quad \theta(t) \quad b_{p}(t) \quad p(t)\right)^{T}
$$

Its dynamics and measurement equations are

$$
\begin{aligned}
& \dot{X}_{2}(t)=\left(\begin{array}{ccccc}
0 & 0 & u(t) & 0 & 0 \\
0 & 0 & r(t) & 0 & 1 \\
0 & -r(t) & 0 & 0 & 0 \\
0 & 0 & 0 & 0 & 0 \\
0 & 0 & 0 & 0 & 0
\end{array}\right) X_{2}(t)+\left(\begin{array}{c}
0 \\
0 \\
q(t) \\
* \\
*
\end{array}\right) \\
& Y_{2}(t)=\left(\begin{array}{ccccc}
1 & 0 & 0 & 0 & 0 \\
0 & 0 & 0 & 1 & 1
\end{array}\right) X_{2}(t)
\end{aligned}
$$


The local observability matrix (2) is calculated below (we omit $t$ for readability):

$$
\begin{aligned}
& \mathbb{O}(t)=\left(\begin{array}{lccc}
Q_{0}(t) & Q_{1}(t) & Q_{2}(t) & Q_{3}(t)
\end{array}\right)\left(\begin{array}{l}
Q_{0}^{T}(t) \\
Q_{1}^{T}(t) \\
Q_{2}^{T}(t) \\
Q_{3}^{T}(t)
\end{array}\right) \\
& =\left(\begin{array}{ccccc}
1 & 0 & 0 & 0 & 0 \\
0 & \left(r^{2}+\dot{r}^{2}\right) u^{2} & \dot{r} u\left(r^{2} u-\ddot{u}\right) & 0 & r \dot{r} u^{2} \\
0 & s y m & -r u \dot{u} & & \\
0 & 0 & u^{2}+\dot{u}^{2} & 0 & r u\left(r^{2} u-\ddot{u}\right) \\
0 & s y m & 0 & 1 & 1 \\
& \multicolumn{2}{c}{u-\ddot{u})^{2}} & & 1 \\
0 & s y m & 1 & 1+r^{2} u^{2}
\end{array}\right)
\end{aligned}
$$

where sym stands for symmetric terms. As previously, thanks to boundedness of the dynamics, the determinant of $\mid \mathbb{O}(t)-$ $s I \mid$ can be lower-bounded by a polynomial $P_{0}(s)$ with $P_{0}(0)=$ $r_{m}^{4} u_{m}^{6}>0$. Then, there exists $\mu>0$ such that $\mathbb{O}(t) \geq \mu I \geq 0$.

This proves that the subset of variables $X_{2}$ is UCO. Again, it can be reconstructed using a Kalman filter. To update the global state $X_{I I}$, we use the following equations

$$
q(t)=q_{m}(t)-b_{q}\left(t_{0}\right), \quad r(t)=r_{m}(t)-b_{r}\left(t_{0}\right)
$$

\section{Observer Synthesis}

\section{1) Observable states:}

Since the systems (7-8), (9-11) and (17-19) are UCO, thanks to Theorem 5, one can construct, for each one, a converging Kalman filter. For each system $S_{i}$, we solve the Riccati equation with $M_{i}$ the state residual covariance matrix for the subset $X_{i}$ (defined in Eq. $(7,9,17)$ respectively)

$$
\dot{M}_{i}(t)=A_{i}(t) M_{i}(t)+M_{i}(t) A_{i}^{T}(t)-M_{i}(t) C_{i}^{T} W_{i}^{-1} C M_{i}(t)+V_{i}
$$

State noises and measurement noises variance matrices are diagonal. For the stationary model,

$$
\begin{aligned}
& W_{0}=\operatorname{diag}\left(\left[\begin{array}{lllllll}
\sigma_{a}^{2} & \sigma_{g}^{2} & \sigma_{g}^{2} & \sigma_{g}^{2} & 0 & 0 & 0
\end{array}\right]\right), \\
& V_{0}=\operatorname{diag}\left(\left[\begin{array}{lllllll}
0 & \sigma_{b}^{2} & \sigma_{b}^{2} & \sigma_{b}^{2} & 0 & 0 & 0
\end{array}\right]\right)
\end{aligned}
$$

for the straight-line motion model,

$$
W_{1}=\operatorname{diag}\left(\left[\begin{array}{ll}
\sigma_{a}^{2} & \sigma_{g}^{2}
\end{array}\right]\right), \quad V_{1}=\operatorname{diag}\left(\left[\begin{array}{llll}
0 & 0 & \sigma_{b}^{2} & \sigma_{q}^{2}
\end{array}\right]\right)
$$

and, for the curve motion model,

$$
W_{2}=\operatorname{diag}\left(\left[\begin{array}{ll}
\sigma_{a}^{2} & \sigma_{g}^{2}
\end{array}\right]\right), \quad V_{2}=\operatorname{diag}\left(\left[\begin{array}{lllll}
0 & 0 & 0 & \sigma_{b}^{2} & \sigma_{p}^{2}
\end{array}\right]\right)
$$

where $\sigma_{g}$ (resp. $\sigma_{a}$ ) is set to the standard error of the gyroscope (resp. the altimeter) and $\sigma_{b}, \sigma_{p}, \sigma_{q}, \sigma_{r}$ are the process noise scaled to cover all uncertainties concerning the dynamics of the biases and of the angular rates.

These three temporally interconnected observers (TIO) will be used one at a time according to a switching policy which we now detail.
2) Switching policy:

In order to determine when to switch from one Kalman filter to another, we need to propagate the whole state $X_{I I}$. Simple thresholds on the estimates of $u$ and $r$ serve to define the switching policy. We also propagate the state residual covariance vector $M_{I I}$.

We define

$M_{I I}=\left[\begin{array}{lllllllll}M_{z} & M_{\phi} & M_{\theta} & M_{p} & M_{q} & M_{r} & M_{b_{p}} & M_{b_{q}} & M_{b_{r}}\end{array}\right]^{T}$

During stops, the $\left[\begin{array}{lllllll}M_{z} & M_{p} & M_{q} & M_{r} & M_{b_{p}} & M_{b_{q}} & M_{b_{r}}\end{array}\right]^{T}$ vector is updated according to Eq. $(23,24)$ and the remaining coordinates of $M_{I I}$ are kept constant.

During the straight-line motion, the $\left[\begin{array}{llll}M_{z} & M_{\theta} & M_{b_{q}} & M_{q}\end{array}\right]^{T}$ vector is updated according to Eq. $(23,25)$ and the remaining coordinates of $M_{I I}$ are updated as follows

$$
\begin{cases}\dot{M}_{\phi}=2 M_{p} & \\ \dot{M}_{p}=-M_{p}^{2} /\left(\sigma_{g}^{2}+M_{b_{p}}\right)+\sigma_{p}^{2}, & \dot{M}_{b_{p}}=\sigma_{b}^{2} \\ \dot{M}_{r}=-M_{r}^{2} /\left(\sigma_{g}^{2}+M_{b_{r}}\right)+\sigma_{r}^{2}, & \dot{M}_{b_{r}}=\sigma_{b}^{2}\end{cases}
$$

During the curve motion, the $\left[\begin{array}{ccccc}M_{z} & M_{\phi} & M_{\theta} & M_{b_{p}} & M_{p}\end{array}\right]^{T}$ vector is updated according to Eq. $(23,26)$ and the remaining coordinates of $M_{I I}$ are updated as follows

$$
\begin{cases}\dot{M}_{q}=-M_{q}^{2} /\left(\sigma_{g}^{2}+M_{b_{q}}\right)+\sigma_{q}^{2}, & \dot{M}_{b_{q}}=\sigma_{b}^{2} \\ \dot{M}_{r}=-M_{r}^{2} /\left(\sigma_{g}^{2}+M_{b_{r}}\right)+\sigma_{r}^{2}, & \dot{M}_{b_{r}}=\sigma_{b}^{2}\end{cases}
$$

\section{3) Simulation results:}

To illustrate the merits of our proposed observer, we report some simulation results. A synthesis model of a 6 DOF rigid body with longitudinal velocity and angular rates as inputs is considered. A representative succession of curves and straight lines is simulated. Measurements are polluted with Gaussian white noises, and pink noises are added to simulate the biases of the gyroscopes. Modeled errors and biases are representative of typical considered low-cost MEMS sensors for automotive applications. In the figures, we note "TIO" values estimated with Temporally Interconnected Observers and refer to debiased values with the term "ZUPT" for Zero Velocity Update which is a reference solution for this problem (see [27] for details).

In Fig. 4, one can see the estimated pitch bias (blue), the real pitch bias (green) and the debiased pitch bias (red).

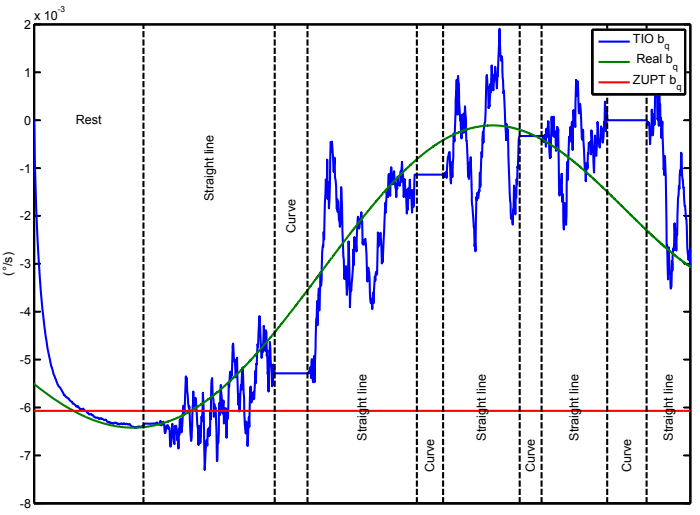

Fig. 4. Estimation of the pitch bias 


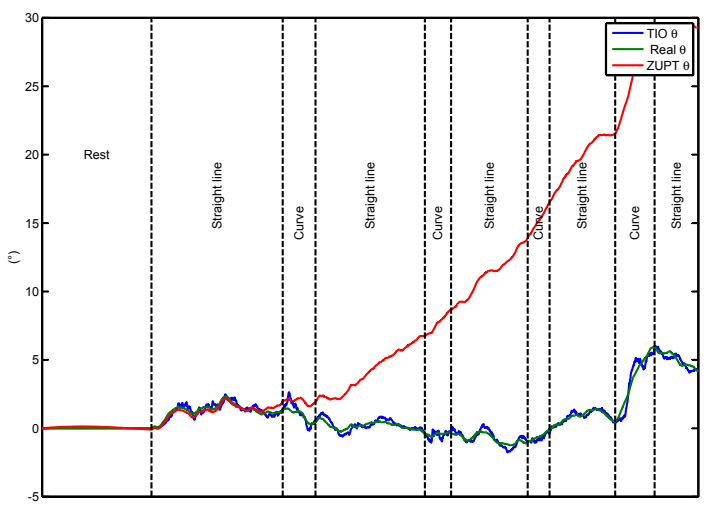

Fig. 5. Estimation of the pitch angle

Debiasing is obtained by a simple technique consisting in removing the mean value of the bias during the stationary phase. At the beginning of the simulation is a stationary phase: the estimated bias converges towards the real bias. After this first phase, one can observe a succession of varying values and constant parts. The parts when the bias estimation varies are straight-line motions during which the pitch bias is observable. It is kept constant in curve motion. The TIO bias is centered around the real bias but remains updated continuously. $^{2}$ The mean error on the value of the bias is divided by 4 between the ZUPT pitch bias and the TIO pitch bias. This is a substantial improvement.

Figure 5 reports comparisons of the TIO pitch angle (blue) and the ZUPT pitch angle (red) to the real pitch angle (green). The mean error is divided by 50 . This vast improvement is essentially due to the altimeter which, as noted in Eq. (14), provides observability on the pitch angle. One clearly sees that TIO does not disturb this estimation in spite of a coupling between roll and pitch angles during curve motion.

In Fig. 6, one can see the TIO roll bias (blue), the real roll bias (green) and the ZUPT roll bias (red). During straightline motion, TIO roll bias is kept constant and its estimate is updated only during curve motion. Each correction step is relatively effective and the estimate of the roll bias is

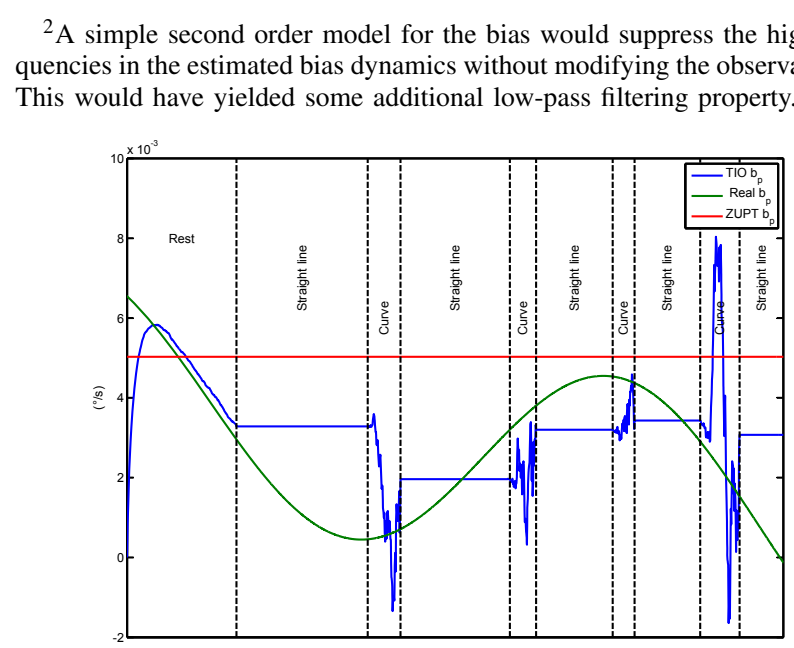

Fig. 6. Estimation of the roll bias

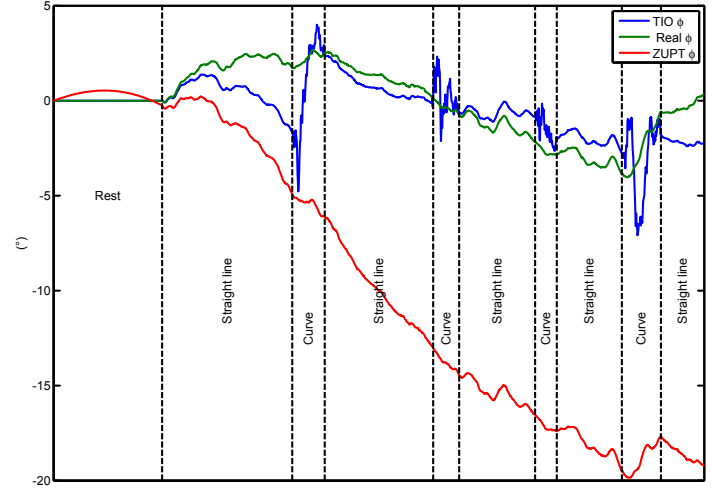

Fig. 7. Estimation of the roll angle

improved after each curve motion. The mean error is divided by 2 .

The benefits of the use of TIO are also emphasized in Fig. 7 where the TIO roll angle (blue), the real roll angle (green) and the ZUPT roll angle (red) are reported. For each straight-line motion, one can see the TIO roll angle becomes erroneous because of the natural variations of the bias. Each curve motion allows to estimate the angle (correction of the value) and the bias (correction of the drift). On overall, with the proposed TIO, the mean error on roll angle is divided by 10 .

\section{CONCLUSION}

An observer for the angular dynamics of an automotive vehicle has been presented under the form of temporally interconnected observers (TIO). This proposed observer design estimates the roll angle during curves and not during straight lines. Beyond the improvement of the estimation of the roll and pitch angles, these TIO permit to determine the projection of the gravity on each accelerometer with a better accuracy. It is then possible to estimate accelerometers biases and, then, to detect faults on the velocimeter. This extra feature (not detailed here for sake of conciseness) improves the reliability of the velocity information, as can be seen in Fig. 8.

As a conclusion, we now present some results obtained on board an actual car which is equipped with a velocimeter, a barometer and MEMS accelerometers and gyroscopes. This setup is exactly the one considered in this study. A GPS receiver is embedded to serve for sake of comparisons only.

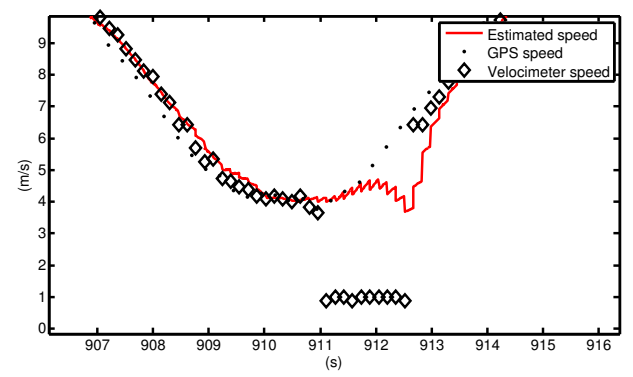

Fig. 8. Estimation of the velocity using complementary information from continuously debiased accelerometers in case of malfunction of the velocimeter 
Figure 8 shows that proposed interconnected and temporally interconnected observers are satisfying. The proposed method allows a significant reduction of the various biases of the sensors. Then, their information can be integrated to determine position estimates. This yields some promising results. In Fig. 9, the trajectory (red) obtained by integration of the estimated velocity with estimated attitudes of the vehicle is presented and compared to the GPS information (blue). After 20 minutes of driving, heading error is less than 15 degrees (without bias correction, it would be approx. 40 degrees) and position error is around 500 meters. These results were obtained using solely the mentioned low-cost sensors: a MEMS altimeter, a velocimeter, MEMS inertial sensors. Interestingly, these results could be improved using map-matching techniques [28]. The computed trajectory is close enough to the actual one to be easily identified using such technique.

\section{Acknowledgement}

The authors wish to thank the SYSNAV team for their technical support and fruitful discussions on this paper.

\section{REFERENCES}

[1] P. Faurre, Navigation inertielle et filtrage stochastique. Dunod, 1971.

[2] M. S. Grewal, L. R. Weill, and A. P. Andrews, Global positioning systems, inertial navigation, and integration. Wiley Inter-science, 2001.

[3] D. Bray, Dynamic Positioning. Oilfield Publications Ltd., 2003.

[4] P. Castillo, A. Dzul, and R. Lozano, "Real-time stabilization and tracking of a four rotor mini rotorcraft," IEEE Trans. Control Systems Technology, vol. 12, no. 4, pp. 510-516, 2004.

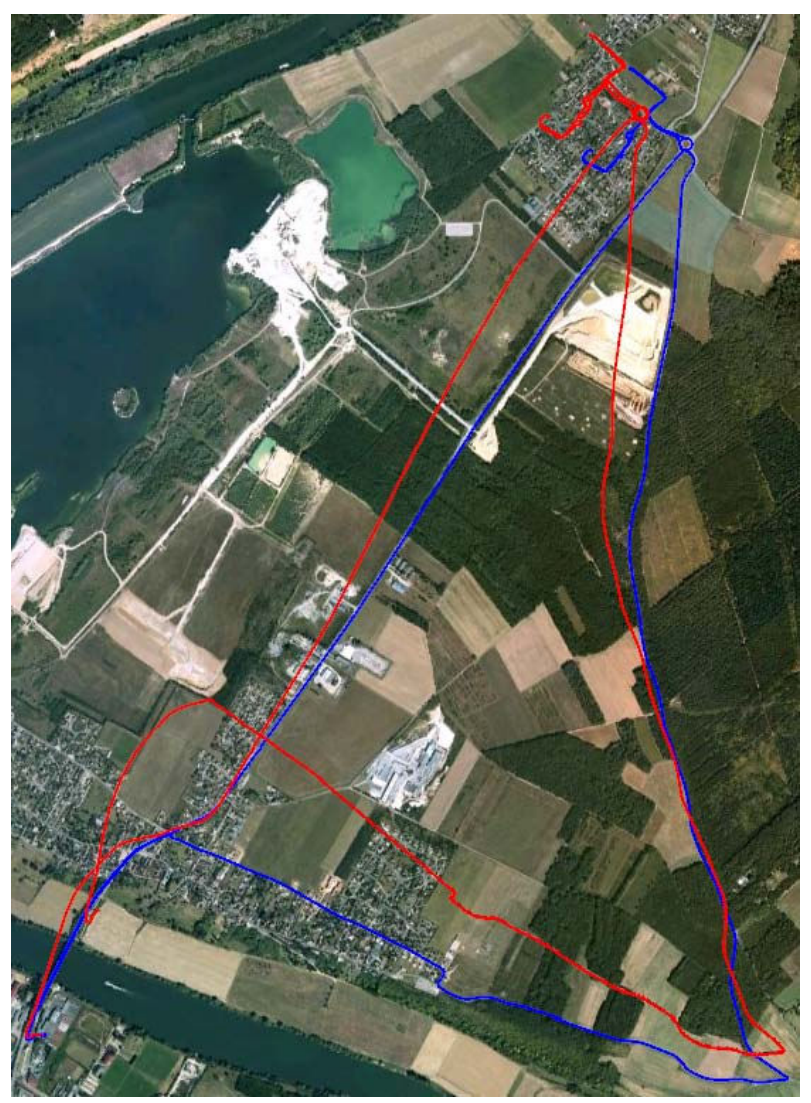

Fig. 9. Comparison of estimated trajectory (red) with GPS (blue)
[5] P.-J. Bristeau, E. Dorveaux, D. Vissière, and N. Petit, "Hardware and software architecture for state estimation on an experimental low-cost small-scaled helicopter," Control Engineering Practice, vol. 18, pp. 733-746, 2010.

[6] G. Dissanayake, S. Sukkarieh, E. Nebot, and H. Durrant-Whyte, "The aiding of a low-cost strapdown inertial measurement unit using vehicle model constraints for land vehicle applications," IEEE Trans. Robotics and Automation, vol. 17, no. 5, pp. 731-747, 2001.

[7] D. Vissière, P.-J. Bristeau, A. P. Martin, and N. Petit, "Experimental autonomous flight of a small-scaled helicopter using accurate dynamics model and low-cost sensors," in Proc. 17th IFAC World Congress, vol. 17, no. 1, 2008.

[8] M. Uliana, F. Andreucci, and B. Papalia, "The navigation system of an autonomous underwater vehicle for antarctic exploration," in Proc. IEEE Marine Technology Society Conference, vol. 1, 1997, pp. 403408.

[9] S.-B. Kim, S.-Y. Lee, T.-H. Hwang, and K.-H. Choi, "An advanced approach for navigation and image sensor integration for land vehicle navigation," in Proc. IEEE 60th Vehicular Technology Conference, vol. 6, 2004, pp. 4075-4078.

[10] I. Skog and P. Handel, "In-car positioning and navigation technologies: a survey," IEEE Trans. Intelligent Transportation Systems, vol. 10, no. 1, pp. 4-21, 2009.

[11] E. Kreindler and P. Sarachik, "On the concepts of controllability and observability of linear systems," IEEE Trans. Automatic Control, vol. 9, no. 2, pp. 129-136, 1964.

[12] L. M. Silverman and H. E. Meadows, "Controllability and observability in time-variable linear systems," SIAM J. Control, vol. 5, no. 1, pp. 64-73, 1967.

[13] R. Hermann and A. Krener, "Nonlinear controllability and observability," IEEE Trans. Automatic Control, vol. 22, no. 5, pp. 728-740, 1977.

[14] A. Gadre, "Observability analysis in navigation systems with an underwater vehicle application," Ph.D. dissertation, Faculty of Virginia Polytechnic Institute and State University, 2007.

[15] G. Besançon and H. Hammouri, "On observer design for interconnectd systems," J. Mathematical Systems, Estimation, and Control, vol. 8, no. 3, pp. 1-25, 1998.

[16] L. Silverman, "Transformation of time-variable systems to canonical (phase-variable) form," IEEE Trans. Automatic Control, vol. 11, no. 2, pp. 300-303, 1966.

[17] J. Willems and S. Mitter, "Controllability, observability, pole allocation, and state reconstruction," IEEE Trans. Automatic Control, vol. 16, no. 6, pp. 582-595, 1971.

[18] M. Ikeda, H. Maeda, and S. Kodama, "Estimation and feedback in linear time-varying systems: A deterministic theory," SIAM J. Control, vol. 13 , no. 2 , pp. 304-326, 1975.

[19] A. Froment, "Commande digitale d'un amortisseur actif," Ph.D. dissertation, Ecole Nationale Supérieure des Mines de Paris, 1984.

[20] R. S. Bucy and P. D. Joseph, Filtering for Stochastic Processes with Applications to Guidance. AMS Chelsea Publishing, 1968.

[21] R. E. Kalman, "Contributions to the theory of optimal control," Boletin de la Sociedad Matematica Mexicana, vol. 5, pp. 102-119, 1960.

[22] L. M. Silverman and B. D. O. Anderson, "Controllability, observability and stability of linear systems," SIAM J. Control, vol. 6, no. 1, pp. $121-130,1968$.

[23] K. S. Tsakalis and P. A. Ioannou, Linear Time-Varying Systems: Control and Adaptation. Prentice Hall, 1993.

[24] T. Kailath, Linear Systems. Prentice Hall, 1980.

[25] G. Besançon, Nonlinear Observers and Applications. Springer, 2007, vol. 363, ch. An Overview on Observer Tools for Nonlinear Systems, pp. 1-33.

[26] R. E. Kalman and R. S. Bucy, "New results in linear filtering and prediction theory," Trans. ASME - J. Basic Eng., vol. 83, pp. 95-108, 1961.

[27] D. A. Grejner-Brzezinska, Y. Yi, and C. K. Toth, "Bridging gps gaps in urban canyons: the benefits of zupts," J. Navigation, vol. 48, no. 4, pp. 217-225, 2001.

[28] P. Davidson, M. Vazquez, and R. Piche, "Uninterrupted portable car navigation system using gps, map and inertial sensors data," in Proc. IEEE 13th International Symposium on Consumer Electronics, 2009, pp. $836-840$. 


\section{APPENDIX}

\section{Proof of the theorem 4}

To a pair $(x, s)$, we associate $x_{s}(t)$ the solution of

$$
\dot{x}_{s}(t)=A(t) x_{s}(t), \quad x_{s}(s)=x
$$

So, we have $x_{s}(t)=\Phi(t, s) x$ and $y_{s}(t)=C(t) x_{s}(t)$.

The function $t \mapsto y_{s}(t)$ verifies $\dot{y}_{s}^{(i)}(t)=Q_{i}^{T}(t) x_{s}(t)$.

Since the system (1) is bounded, there exist $(a, c) \in \mathbb{R}$ such that

$$
|A(t)| \leq a \quad, \quad\left|Q_{i}(t)\right| \leq c \quad \forall t, \forall i \in\{0, m\}
$$

It means that, for all $(s, t)$,

$$
\begin{array}{r}
\exp (-a|t-s|) \leq|\Phi(t, s)| \leq \exp (a|t-s|) \\
\left|y_{s}(t)\right| \leq c \exp (a|t-s|)|x|
\end{array}
$$

First, consider the Taylor approximation with integral form of the remainder term applied to $y_{s}(t)$

$$
\begin{gathered}
y_{s}(t)=\mathcal{P}_{S}(t-s)+\mathcal{R}_{s}(t, s) \\
\text { with } \mathcal{P}_{s}(t-s)=\sum_{i=0}^{m} \frac{(t-s)^{i}}{i !} y_{s}^{(i)}(s) \\
\text { and } \mathcal{R}_{S}(t, s)=\int_{s}^{t} \frac{(t-r)^{m}}{m !} y_{s}^{(m+1)}(r) d r
\end{gathered}
$$

One has

$$
\begin{gathered}
\int_{-\infty}^{s} \exp (-\lambda[s-t])\left|y_{s}(t)\right|^{2} d t \geq T_{\mathcal{P}}-T_{\mathcal{R}} \\
\text { where } T_{\mathcal{P}}=\frac{1}{2} \int_{-\infty}^{s} \exp (-\lambda[s-t])\left|\mathcal{P}_{S}(t-s)\right|^{2} d t \\
T_{\mathcal{R}}=\int_{-\infty}^{s} \exp (-\lambda[s-t])\left|\mathcal{R}_{s}(t, s)\right|^{2} d t
\end{gathered}
$$

It is desired to find a lower bound for $T_{\mathcal{P}}$

$$
\begin{aligned}
T_{\mathcal{P}} & =\int_{-\infty}^{s} \exp (-\lambda[s-t])\left|\sum_{i=0}^{m} \frac{(t-s)^{i}}{i !} y_{s}^{(i)}(s)\right|^{2} d t \\
& =\frac{1}{\lambda} \int_{-\infty}^{0} \exp (\tau)\left|\sum_{i=0}^{m} \frac{\tau^{i}}{i !} \frac{y_{s}^{(i)}(s)}{\lambda^{i}}\right|^{2} d \tau
\end{aligned}
$$

One can see that $T_{\mathcal{P}}$ is a non negative quadratic form in $y_{s}(s)$, $\ldots, y_{s}^{(m)}(s)$ :

$$
T_{\mathcal{P}}=\frac{1}{\lambda}\left(\begin{array}{llll}
y_{s}^{T}(s) & \frac{y_{s}^{(1) T}(s)}{\lambda} & \ldots & \frac{y_{s}^{(m) T}(s)}{\lambda^{m}}
\end{array}\right) \Xi\left(\begin{array}{c}
y_{s}(s) \\
\frac{y_{s}^{(1)}(s)}{\lambda} \\
\vdots \\
\frac{y_{s}^{(m)}(s)}{\lambda^{m}}
\end{array}\right)
$$

where the matrix $\Xi$ only depends of $m$. Moreover, the integral $T_{\mathcal{P}}$ is null if and only if all the coefficients of the polynom in $\tau$ under the integral are null, that is to say, if and only if all the components of all the derivatives $y_{s}^{(i)}(s)$ are null. So the matrix $\Xi$ is positive definite and there exists $\alpha>0$ such that:

$$
T_{\mathcal{P}} \geq \frac{\alpha}{\lambda^{2 m+1}} \sum_{i=0}^{m}\left|y_{s}^{(i)}(s)\right|^{2}
$$

Otherwise, one has

$$
x^{T} \mathbb{O}(s) x=\left(\begin{array}{lll}
y_{s}(s) & \ldots & \dot{y}_{s}^{(m)}(s)
\end{array}\right)\left(\begin{array}{c}
y_{s}^{T}(s) \\
\vdots \\
\dot{y}_{s}^{(m) T}(s)
\end{array}\right)=\sum_{i=0}^{m}\left|y_{s}^{(i)}(s)\right|^{2}
$$

So, with Eq. (2), one can conclude

$$
T_{P} \geq \frac{\alpha \mu}{\lambda^{2 m+1}}|x|^{2}
$$

Concerning the term $T_{\mathcal{R}}$,

$$
T_{\mathcal{R}}=\frac{1}{\lambda} \int_{-\infty}^{0} \exp (\tau)\left|\mathcal{R}_{s}\left(\frac{\tau}{\lambda}+s, s\right)\right|^{2} d \tau
$$

with

$$
\begin{aligned}
\mathcal{R}_{s} & \left(\frac{\tau}{\lambda}+s, s\right)=\int_{s}^{\frac{\tau}{\lambda}+s} \frac{\left(\frac{\tau}{\lambda}+s-r\right)^{m}}{m !} y_{s}^{(m+1)}(r) d r \\
& =\frac{1}{\lambda^{m+1}} \int_{0}^{\tau} \frac{\rho^{m}}{m !} y_{s}^{(m+1)}\left(s+\frac{\tau-\rho}{\lambda}\right) d \rho
\end{aligned}
$$

From Eq. (30), one deduces that

$$
\begin{aligned}
\left|\mathscr{R}_{S}\left(\frac{\tau}{\lambda}+s, s\right)\right| & \leq \frac{1}{\lambda^{m+1}} \frac{c}{m !}|x| \int_{0}^{|\tau|} \rho^{m} \exp \left(a \frac{|\tau-\rho|}{\lambda}\right) d \rho \\
& \leq \frac{1}{\lambda^{m+1}} \frac{c}{(m+1) !}|\tau|^{m+1} \exp \left(a \frac{|\tau|}{\lambda}\right)|x|
\end{aligned}
$$

So, one can find an upper bound to $T_{\mathcal{R}}$

$$
T_{\mathcal{R}} \leq \frac{1}{\lambda^{2 m+3}} \frac{c^{2}}{[(m+1) !]^{2}}|x|^{2} \int_{-\infty}^{0} \exp (\tau) \tau^{2(m+1)} \exp \left(2 a \frac{|\tau|}{\lambda}\right) d \tau
$$

For any $\lambda \in[2 a+\varepsilon, \infty)$ with $\varepsilon>0$, the integral is bounded independently of $\lambda$, so there exists $\beta>0$ such that

$$
T_{\mathcal{R}} \leq \frac{\beta}{\lambda^{2 m+3}}|x|^{2}
$$

Combining the equations (31) and (32), one obtains

$$
\begin{aligned}
\int_{-\infty}^{s} \exp (-\lambda[s-t])\left|y_{s}(t)\right|^{2} d t & \geq \frac{\alpha \mu}{2 \lambda^{2 m+1}}|x|^{2}-\frac{\beta}{\lambda^{2 m+3}}|x|^{2} \\
& \geq \frac{\alpha \mu \lambda^{2}-2 \beta}{\lambda^{2 m+3}}|x|^{2}
\end{aligned}
$$

where $\eta=\frac{\alpha \mu \lambda^{2}-2 \beta}{\lambda^{2 m+3}}$ is strictly positive for all $\lambda$ sufficiently large in $[2 a+\varepsilon, \infty)$.

For all $S \geq 0$, we have

$$
\begin{aligned}
\int_{-\infty}^{s} \exp (-\lambda[s-t])\left|y_{s}(t)\right|^{2} d t & \leq \int_{s-S}^{s}\left|y_{s}(t)\right|^{2} d t \\
& +\frac{c^{2}}{\lambda-2 a} \exp (-(\lambda-2 a) S)|x|^{2}
\end{aligned}
$$

We can choose $S=\frac{\log \left(\frac{2 c^{2}}{[\lambda-2 a \mid \eta}\right)}{\lambda-2 a}$, thus

$$
\int_{s-S}^{s}\left|y_{s}(t)\right|^{2} d t \geq \frac{\eta}{2}|x|^{2} \forall(x, s)
$$

Now,

$$
\int_{s-S}^{s}\left|y_{S}(t)\right|^{2} d t=x^{T} W^{*}(s-S, s) x
$$

In summary, we have constructed $\mathrm{S}$ such that for all $(x, s), W^{*}(s-S, s) \geq a_{1}(S) I>0$. We apply Theorem 2 and conclude that the system is UCO. 